\title{
THE NATURE OF \\ THE NEONATAL PULMONARY HYALINE MEMBRANE
}

BY

\author{
R. A. BARTER and T. G. MADDISON \\ From the Royal Women's Hospital, Melbourne
}

(RECEIVED FOR PUBLICATION DECEMBER 21, 1959)

Hyaline membranes are seen in the lungs of nearly one-third of prematurely born infants who die after birth at the Royal Women's Hospital, Melbourne. The nature of the membranes has been discussed often in the literature (Farber and Wilson, 1932; Tregillus, 1951; Claireaux, 1953; Bruns and Shields, 1954; Gilmer and Hand, 1955; Lendrum, 1955; Lynch and Mellor, 1955; Stevenson and Laufe, 1955; Gitlin and Craig, 1956 and many others). The subject has also been reviewed by Tran-Dinh-De and Anderson (1953), Curtis (1957), and others, and in an Editorial in the Lancet (1958). So far there is no generally accepted explanation of the mode of origin of the membranes and opinions differ widely on the nature of the material of which they are composed. Two main views are held. One postulates an origin from the contents of the amniotic sac and the other from an exudate from the pulmonary capillaries.

The present study is based on observation of morphological changes in affected lungs and was undertaken after a variety of histochemical and interferometric techniques had been employed without significant result. From microscopic examination of the lungs using sections prepared by conventional methods it is concluded that hyaline membranes result from necrosis of epithelial cells in respiratory bronchioles and form in situ.

\section{Material}

Sections of the lungs have been examined from 133 consecutive cases of hyaline membrane disease occurring in a three-year period and the clinical notes of each case have been reviewed. Necropsy is routine for infants who die and only very rarely is objection made. Approximately 320 autopsies (stillbirths and neonatal deaths) are performed each year. Blocks are taken from the lower lobe of one lung in every case, fixed in formalin and prepared for microscopic examination in the usual way. Sections are cut from one block in each case.
Microscopic Findings. Most hyaline membranes are of a uniform eosinophilic and homogeneous appearance and line air spaces which appear to have been patent at the time of death. The affected air spaces are usually described as alveolar ducts and sometimes as alveolar ducts and alveoli. It is now clear that the alveolar components of the lungs are not involved in hyaline membrane disease and that respiratory bronchioles are the site of hyaline membrane formation.

In sections stained to show elastic fibres the characteristic pattern for respiratory bronchioles is seen around all hyaline membrane affected spaces (Fig. 1). Between affected spaces the lung has a solid structure in which elastic fibres are less frequent, stain faintly and are of irregular distribution. Frequently hyaline membranes are seen lining one side of a respiratory bronchiole while the opposite side is lined normally by cuboidal epithelial cells. In some cases hyaline membranes are seen in longitudinal sections of respiratory bronchioles abutting on the normal columnar lining of terminal bronchioles. Spaces lined by membranes usually have a surrounding lamina propria outside which there are obvious respiratory structures of smaller dimension which are not patent. These are the definitive alveolar tissues in which hyaline membranes do not occur.

In the immature lung of the 1,001-1,500 $\mathrm{g}$. baby the general anatomical features seem to us to be as illustrated in Fig. 2.

$T$ is a terminal bronchiole around which the elastic layer is continuous. $\quad R_{1}$ and $R_{2}$ are respiratory bronchioles around which the elastic layer is interrupted. $A$ is a site containing potential alveolar tissues which may or may not be functional at this stage of development. Here the elastic fibres have no pattern and are less numerous.

The transition from a terminal to a respiratory bronchiole is abrupt. The columnar cells of the terminal bronchiole give way to much less con- 


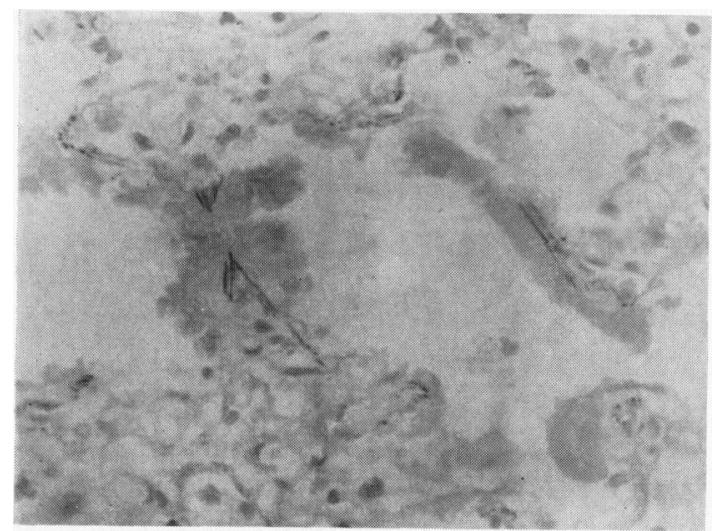

FIG. 1.-Section of respiratory bronchiole showing interrupted pattern of elastic fibres.

(Weigert's elastic stain $\times 228$.)

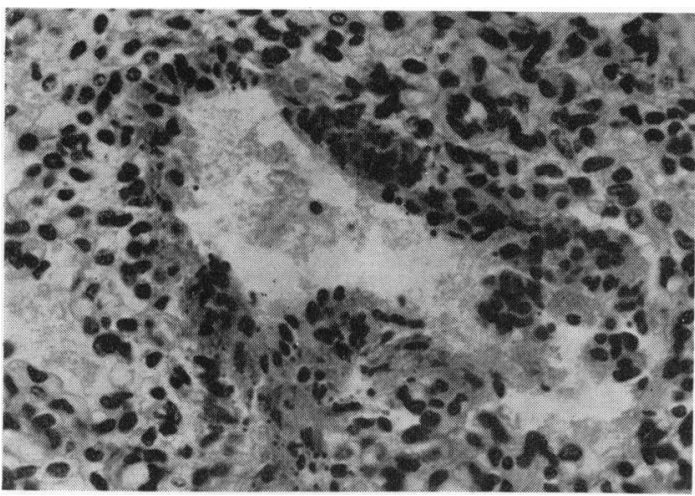

FIG. 3.-Section of respiratory bronchiole lined by partly necrotic epithelial cells.

(H. and E. $\times$ 228.)

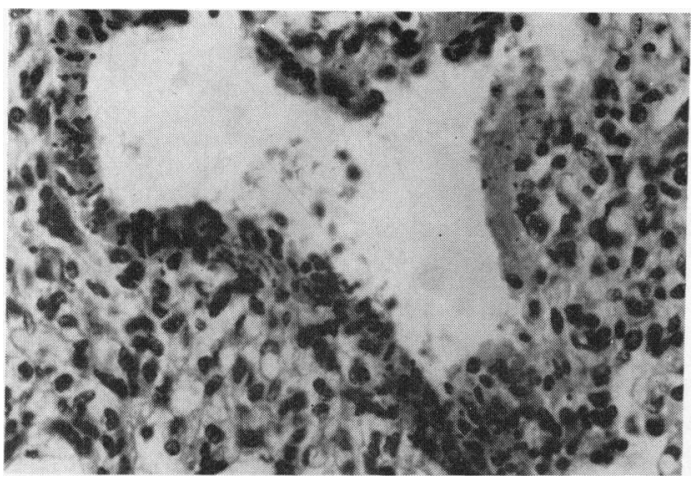

FIG. 4.-Section of respiratory bronchiole showing partly necrotic epithelial cells and a segment of hyaline membrane on the right. (H. and E. $\times 228$.)

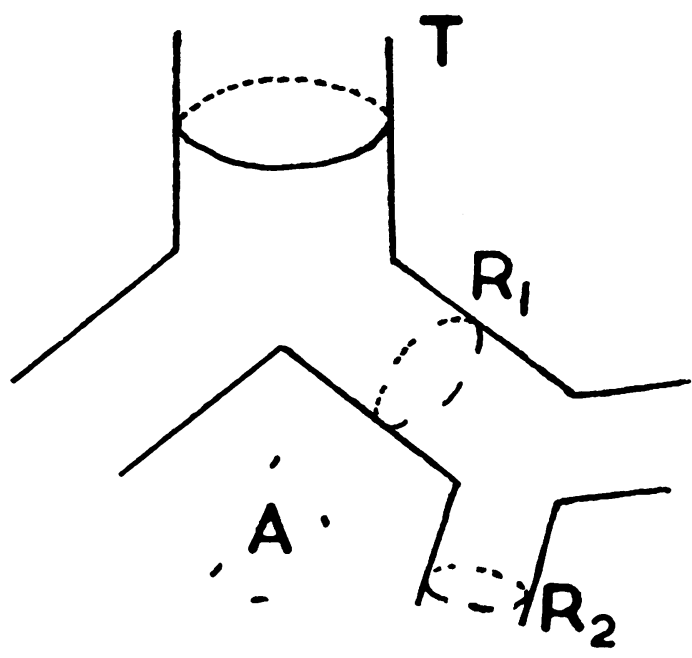

FIG. 2.-Diagrammaticillustration of elastic fibres around bronchioles.

spicuous epithelial cells which are cuboidal or lower. The elastic layer becomes interrupted and the lumen is narrower. The cuboidal cells lining respiratory bronchioles have a clear colourless cytoplasm and a centrally placed round vesicular nucleus.

In $69 \%$ of the cases in this series the membranes were not all entirely eosinophilic but contained basophilic particles. In some cases these stained deeply while in others they were just visible. Sometimes the origin of the basophilic particles from the nuclei of epithelial cells was obvious (Fig. 3) mainly because of the regular distribution around the lumen and also because of the size and location of the material in relation to the surrounding eosinophilic membrane. Except when the particles were extremely fragmented there was no possibility of mistaking the appearances for degenerate leucocytes. Appreciation of the epithelial origin of the lesion depicted in Fig. 4 led to detailed examination of all sections, membrane by membrane, in order to follow the changes by which membranes result from degeneration of epithelial cells.

The first changes observed occur simultaneously in cytoplasm and nucleus. The cytoplasm swells slightly and becomes eosinophilic and the nucleus loses its vesicular appearance and soon becomes pyknotic (Fig. 5). Later there is an enormous increase in the size of the cell and an increase in eosinophilia. The nucleus elongates or fragments and is either passed out of the cell or blends with the cytoplasm. Finally, distinction between the cells becomes difficult except on the luminal aspect of the membrane which is somewhat irregular. This 


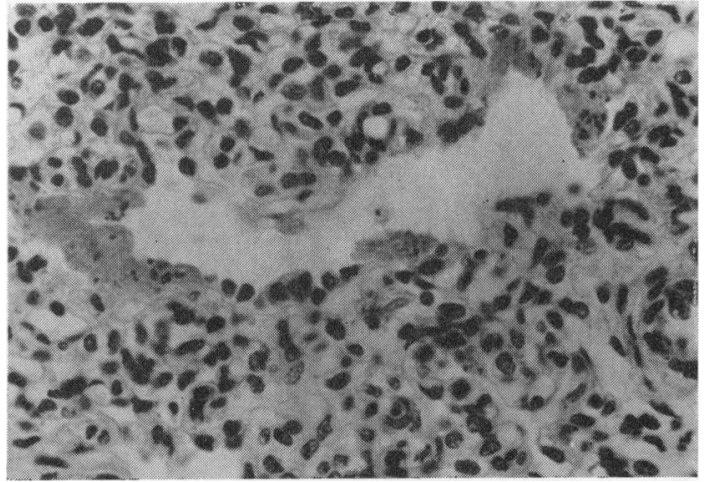

FIG. 5.-Section of respiratory bronchiole showing intact epithelial cells flanked by hyaline membrane. Some nuclei are pyknotic and others in the membrane are fragmented. (H. and E. $\times 228$.)

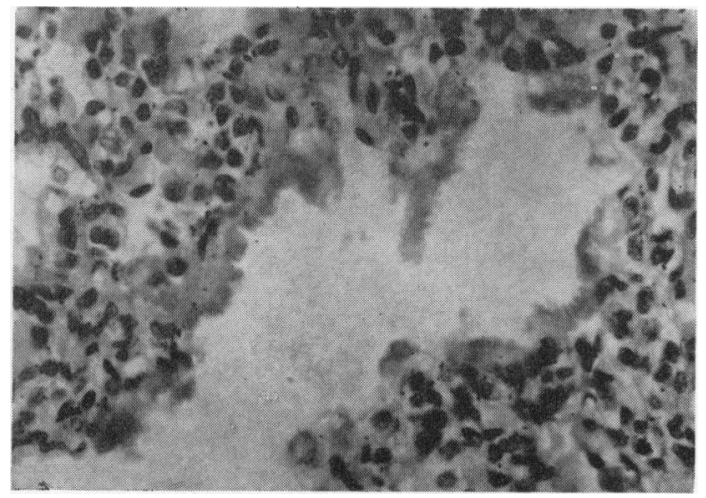

FIG. 6.-Section of respiratory bronchiole showing typical hyaline membrane and characteristic irregular edge. $(\mathrm{H}$ and $\mathrm{E} . \times 228$.)

irregularity often accurately reflects the margins of the original cells (Fig. 6).

\section{Clinical Features}

The 133 cases resulted from 121 pregnancies. There were 67 male and 66 female infants. Nearly all died between two and 48 hours after birth (Fig. 7) and the majority died in the first 16 hours.

One hundred and twenty-three infants were premature, 10 weighed over $2,500 \mathrm{~g}$. and one of these was postmature (Fig. 8). Infants below $1,000 \mathrm{~g}$. showed pulmonary hyaline membranes, but the majority of the cases occurred in the 1,001 2,000 g. range.

The incidence of the lesion is inversely proportional to the gestational age and $60 \%$ were asphyxiated at birth (Fig. 9). Asphyxia is defined as taking two minutes or more to establish rhythmical respirations after delivery and most of these infants received lobeline and/or coramine. In $60 \%$ of cases it was recorded that oxygen was administered at delivery, usually by an intranasal catheter. This is a common procedure in our hospital for prematurely born infants and the actual number so treated is higher than recorded. Asphyxia at birth was more common in infants born at 34 weeks and over $(76 \%)$ than in the group under 34 weeks ( $54 \%)$.

Of the 24 infants delivered abdominally, 20 (83\%) were asphyxiated at birth and usually severely. This incidence of asphyxia is higher than for the series as a whole and suggests that it is asphyxia in cases delivered by caesarian section and not this mode of delivery per se which is the important factor in the subsequent development of hyaline membrane disease. In $32 \%$ of cases delivery was by breech.

The complications of pregnancy, apart from maternal diabetes, were those associated with premature delivery although the individual incidences were greater than expected in premature deliveries as a whole (Table 1). There were 24 multiple pregnancies. In 11 (10 twin, one triplet), all the infants died and showed hyaline membranes at autopsy. In 12 multiple pregnancies (11 twin, one triplet), only the second twin or third triplet was affected.

Nearly one half of the infants who died in the 1,001-2,000 g. group showed hyaline membrane formation (Table 2) but the maximum incidence occurred in the 1,001-1,500 g. group.

In $61 \%$ of the cases pulmonary hyaline membranes were the only major findings at necropsy.

The condition of the infants was poor from birth and signs of respiratory distress developed rapidly. In most infants there was no period of well-being. Lateral chest and lower sternal retraction occurred early and became progressively more severe. An expiratory grunting cry was easily heard. The four limbs often assumed an abducted and externally rotated position. Apnoeic attacks with cyanosis occurred with increasing severity. On auscultation

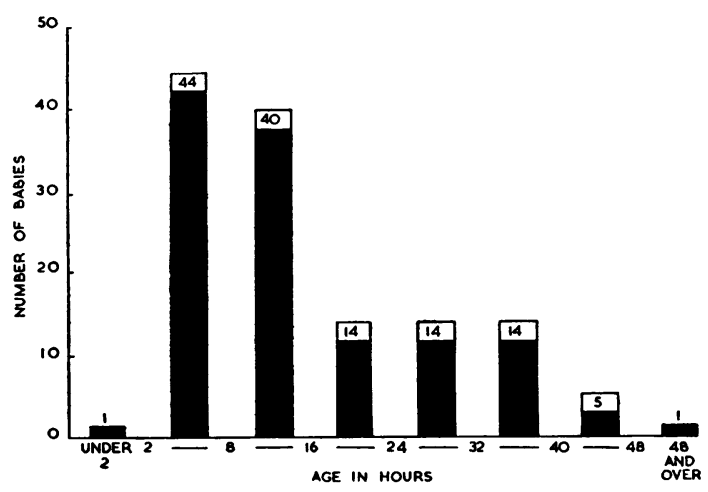

Fig. 7.-Age of infants at death. 


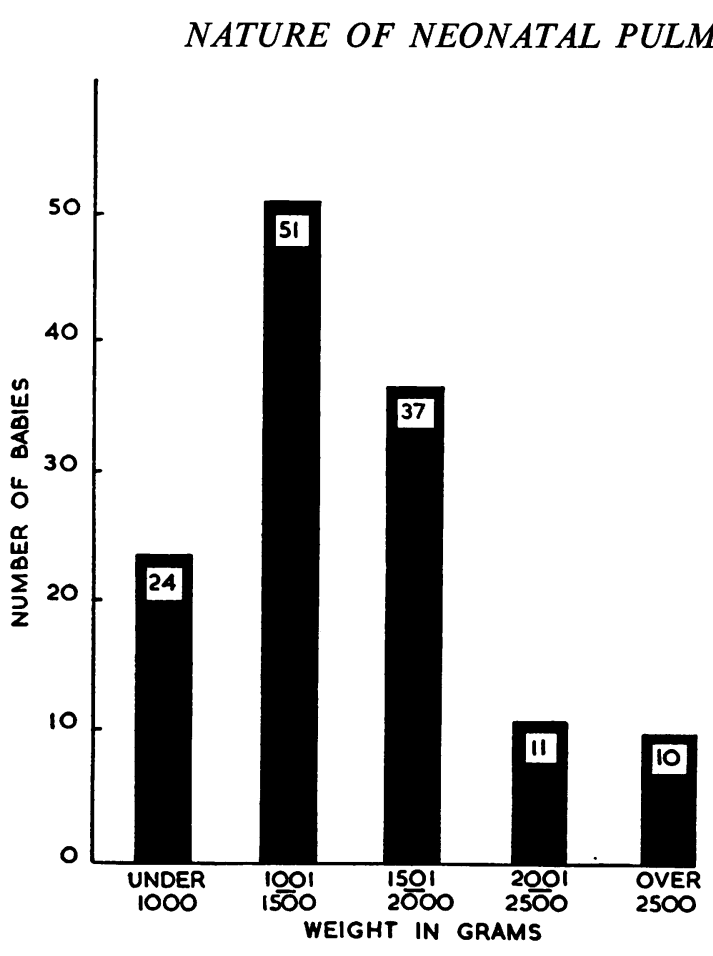

FIG. 8.-Birth weight.

the only constant finding was poor air entry into the lungs.

\section{Discussion}

The origin of hyaline membranes in infants from necrosis of epithelial cells in respiratory bronchioles

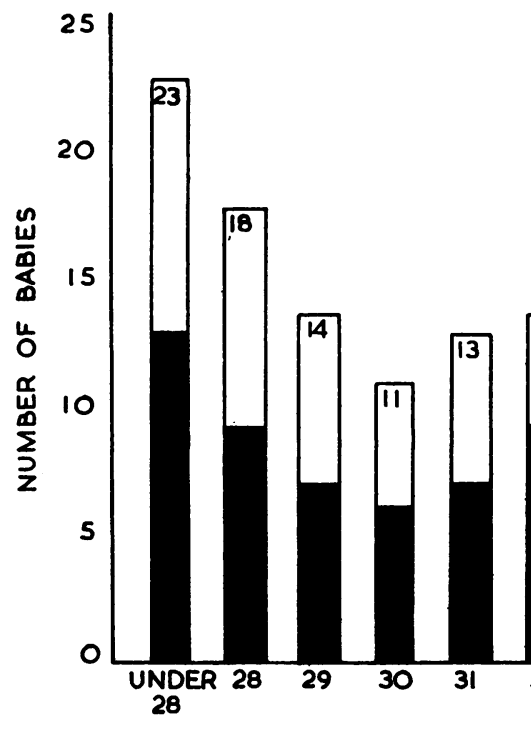

TABLE 1

COMPLICATIONS OF PREGNANCY

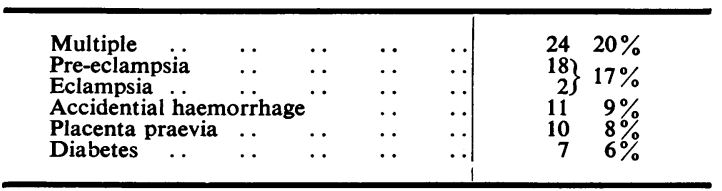

TABLE 2

BIRTH WEIGHT, NEONATAL DEATHS AND HYALINE MEMBRANE INCIDENCE

\begin{tabular}{c|c|c|c}
\hline \multirow{2}{*}{$\begin{array}{c}\text { Weight } \\
\text { (g.) }\end{array}$} & $\begin{array}{c}\text { Neonatal } \\
\text { Deaths }\end{array}$ & Hyaline Membrane \\
\cline { 3 - 4 } & & Nos. & $\begin{array}{c}\text { Incidence } \\
\text { per 1,000 } \\
\text { Live Births }\end{array}$ \\
\hline Up to 1,000 & 184 & 24 & 125 \\
$1,001-1,500$ & 122 & 51 & 204 \\
$1,501-2,000$ & 71 & 37 & 78 \\
$2,001-2,500$ & 47 & 11 & 11 \\
Over 2,500 & 96 & 10 & 0.5 \\
\hline
\end{tabular}

has not been so described until recently (Barter, 1959). Tregillus (1951) showed lesions in the epithelial linings of terminal bronchioles but did not state clearly the relationship of such lesions to hyaline membranes; nor did he appear to appreciate that the principal lesion is confined to respiratory bronchioles. Lynch and Mellor (1955) using histochemical methods have hinted at a relationship between membranes and bronchiolar epithelial cells but so far have come to no definite conclusions about the nature of the disease. However, they have shown similar histochemical properties between epithelial cells in the respiratory tract and hyaline membranes.

Recognition of epithelial necrosis in terminal bronchioles contributed largely to our understanding of the process in respiratory bronchioles. The changes observed in an occasional terminal bronchiole are obviously those of degeneration in epithelial cells and do not occur except when hyaline membranes are present in respiratory bronchioles. The fact that nuclear material is observed in some membranes in $69 \%$ of cases led to the investigation of cases showing minimal membrane formation.

FIG. 9.-Gestational age; $\boldsymbol{D}=$ number asphyxiated at birth. 
All stages of epithelial necrosis from nearly intact cells to those containing faint remnants of nuclei can be observed in many of these cases. That these are the nuclear remnants of epithelial cells is evident from studying all the phases of the degenerative process. It has become clear that epithelial cell necrosis is the basis of hyaline membrane formation and that lesions are found in both terminal bronchioles and respiratory bronchioles but mainly in the latter. The epithelial linings of the trachea and bronchi are not affected and we have found no evidence that membranes form in alveolar ducts or alveoli. Furthermore, it seems likely from our own unpublished observations that alveolar tissues are not as well developed in the premature lung as commonly implied.

Much of the controversy at present about the nature of hyaline membranes centres on the assumption that the material present is derived from substances in the air spaces or from the pulmonary capillaries. Considerable evidence has been presented in favour of one or both explanations. Farber and Sweet (1931) first proposed an origin from the contents of the amniotic sac and changed the name of the lesion, hyaline membrane (Johnson and Meyer, 1925) to vernix membrane. Claireaux (1953) published the results of experimental studies in favour of an origin from aspiration of epidermal material. More recently it has been suggested that thromboplastic substances from the liquor aid in the formation of fibrin from an exudate from the capillaries (Laufe and Stevenson, 1954).

Emphasis has been placed on the exudate hypothesis by the claims of Gitlin and Craig (1956) that membranes are composed largely of fibrin. These workers used a fluorescin labelled antibody for fibrin and showed that membranes in treated sections were brightly fluorescent. Confirmation of the presence of fibrin has been afforded by van Breemen, Neustein and Bruns (1957) who studied thin sections of membranes with an electron microscope. Brown (1959) has stated that the evidence for fibrin is incontrovertible. It should be realized, however, that neither of the techniques proves that the membrane consists of nothing but fibrin. The presence of some of it is not disputed, but we believe that it results from exudation after loss of the normal epithelial lining and is only a small component of the membrane.

The reported incidence of hyaline membranes from different centres shows some variation but there is a remarkable difference between that in South India (Hadley, Gault and Graham, 1958) or Vietnam (Tran-Dinh-De, 1959) and, for example, Melbourne. Thus Hadley et al. found only one case in 215 neonatal deaths and from discussion with Tran-Dinh-De we estimate our incidence to be some 20 times greater than his. At present there is no explanation for these differences.

Experimental methods have not reproduced the disease in a manner corresponding to its occurrence in the human. Bruns and Shields (1954) have, however, produced what are apparently hyaline membranes in the guinea-pig lung after prolonged exposure to oxygen. These workers state that the membranes form as a result of epithelial necrosis in bronchioles. Their observations on the derivation of membranes so produced is in accord with our own in premature infants.

It is attractive to suggest that a noxious substance is the cause of the necrosis in this disease but the evidence for oxygen poisoning in the human premature infant, exposed for short periods, is incomplete.

\section{Summary}

Detailed microscopic study of the lungs of premature infants affected by hyaline membrane disease shows that the membranes result from epithelial cell necrosis in respiratory bronchioles and form in situ.

A description of 133 cases of hyaline membrane disease including clinical features is presented.

\section{REFERENCES}

Barter, R. A. (1959). The neonatal pulmonary hyaline membrane. Lancet, 2. 160.

Brown, R. J. K. (1959). Respiratory difficulties at birth. Brit.

Bruns, P. D. and Shields, L. V. (1954). High oxygen and hyalinelike membranes. Amer. J. Obstet. Gynec., 67, 1224.

Claireaux, A. E. (1953). Hyaline membrane in the neonatal lung. Lancet, 2, 749.

Curtis, P. (1957). Hyaline membrane disease. J. Pediat., 51, 726.

Farber, S. and Sweet, L. K. (1931). Amniotic sac contents in the lungs of infants. Amer. J. Dis. Child., 42, 1372.

and Wilson, J. L. (1932). The hyaline membrane in the lungs. Arch. Path. (Chicago), 14, 437.

Gilmer, W. S. and Hand, A. M. (1955). Morphological studies of hyaline membranes in the newborn infant. A.M.A. Arch. Path., 59, 207.

Gitlin, D. and Craig, J. M. (1956). The nature of the hyaline membrane in asphyxia of the newborn. Pediatrics, 17, 64.

Hadley, G. G., Gault, E. W. and Graham, M. D. (1958). A study of pathology of stillbirths and neonatal deaths in South India. J. Pediat., 52, 139.

Johnson, W. C. and Meyer, J. R. (1925). A study of pneumonia in the stillborn and newborn. Amer. J. Obstet. Gynec., 9, 151.

Lancet (1958). Editorial: Hyaline membrane. 2, 945.

Laufe, L. E. and Stevenson, S. S. (1954). Pulmonary hyaline membranes. Preliminary report on experimental production. Obstet. and Gynec., 3, 637.

Lendrum, F. C. (1955). The 'pulmonary hyaline membrane' as a manifestation of heart failure in the newborn infant. J. Pediat., 47, 149.

Lynch, M. J. G. and Mellor, L. D. (1955). Hyaline membrane disease of newborn premature lungs: A new approach. Ibid., 47,275 .

Stevenson, S. S. and Laufe, L. E. (1955). Experimental production of the pulmonary hyaline membrane syndrome. Ibid., 47, 40 .

Tran-Dinh-De (1959). Personal communication.

- and Anderson, G. W. (1953). Hyaline-like membranes associated with diseases of the newborn lungs: $A$ review of the literature. Obstet. gynec. Surv., 8, 1 .

Tregillus, J. (1951). The asphyxial membrane in the lungs of liveborn infants. J. Obstet. Gynaec. Brit. Emp., 58, 406.

van Breemen, V. L., Neustein, H. B. and Bruns, P. D. (1957). Pulmonary hyaline membranes studied with the electron microscope. Amer. J. Path., 33, 769. 